\title{
Enquête
}

Archives de la revue Enquête

6 | 1991

La socialisation de la jeunesse

\section{Comportements de chahut et performances scolaires des filles et des garçons au collège}

\section{Georges Félouzis}

\section{(2) OpenEdition}

Journals

Édition électronique

URL : http://journals.openedition.org/enquete/140

DOI : $10.4000 /$ enquete. 140

ISSN : 1953-809X

Éditeur :

Cercom, Éditions Parenthèses

Édition imprimée

Date de publication : 2 juin 1991

\section{Référence électronique}

Georges Félouzis, «Comportements de chahut et performances scolaires des filles et des garçons au collège », Enquête [En ligne], 6 | 1991, mis en ligne le 27 juin 2013, consulté le 19 avril 2019. URL:

http://journals.openedition.org/enquete/140; DOI : 10.4000/enquete.140 


\title{
Comportements de chahut et performances scolaires des filles et des garçons au collège
}

\author{
Georges Félouzis
}

1 La sociologie de la reproduction qui «traite le rapport pédagogique comme un simple rapport de communication» (Bourdieu \& Passeron, 1970) montre que la maîtrise différentielle des codes linguistiques propres au langage scolaire est au principe de la sursélection des enfants d'origine ouvrière par rapport aux enfants de cadres.

2 Mais la possession d'un capital linguistique plus ou moins important ne peut rendre compte de la réussite différentielle des élèves selon le sexe. En effet, quelle que soit l'origine sociale, les filles ont un taux de réussite scolaire supérieur aux garçons, et ceci de l'école primaire jusqu'au baccalauréat (Cherkaoui, 1982 ; Establet, 1987).

3 Nous voudrions tenter ici une hypothèse explicative de ces différences de réussite scolaire entre les sexes en poussant plus avant l'hypothèse de $P$. Bourdieu et J.-C Passeron: le rendement (l'efficacité) de la communication pédagogique dépend, certes, de la maîtrise des codes linguistiques par les récepteurs, mais pas seulement de celle-ci. La communication pédagogique est non seulement un rapport de communication, mais aussi un rapport d'interaction pour lequel l'émetteur et le(s) récepteur(s) sont, dans la plupart des cas, directement en présence dans un même lieu clos (la classe). Ceci implique des codes comportementaux dont la maîtrise différentielle selon le sexe peut rendre compte des différences de rendement de la communication pédagogique, et donc des différences de réussite scolaire pour les filles et les garçons ${ }^{1}$.

4 Cette plus ou moins grande maitrise des codes comportementaux peut être mesurée par la négative, c'est-à-dire par la propension, pour chaque sexe, à perturber la communication pédagogique par des comportements de chahut. Il nous faut donc définir ce qu'est le chahut et comment en distinguer les différentes formes. La distinction entre un chahut traditionnel, marquant l'intégration des élèves à la classe et leur intériorisation des normes scolaires, et un chahut anomique, plus individuel, exprimant un refus de l'école et de ces normes (Testanière, 1967b), montre que seule la forme 
anomique du chahut peut exprimer une non-maitrise ou un non-respect des codes comportementaux propre à l'espace scolaire. Les observations menées au cours du travail de terrain ne nous ont pas permis d'observer de chahut collectif digne d'une «anthologie» tel qu'en décrit J. Testanière. Au contraire, le chahut observé dans les classes est, dans la plupart des cas, individuel : ceci nous autorise donc à le définir comme une forme de refus de l'école et comme perturbation de la communication pédagogique exprimant une non-maîtrise ou une non-acceptation des codes interactifs de la classe.

\section{Appréhension et mesure des comportements de chahut}

5 L'appréhension et la mesure des comportements de chahut se sont faites par l'observation directe des comportements à l'aide d'une caméra vidéo installée dans les salles de classe. Le problème posé par la présence de la caméra est celui de l'effet sur les comportements observés. Comme le souligne N. Ramognino (1989), « l'enregistrement des données transforme et dé-forme la "réalité" sans que l'observateur ne puisse la contrôler et maîtriser, sinon dans un déplacement de ces propres questions ». Conscient de cette influence de la situation d'enquête sur les comportements des élèves, nous nous sommes efforcé de limiter le plus possible les réactions d'exubérance et de blocage que pouvait susciter le dispositif d'enquête (une caméra, un pied, un magnétoscope portatif) sur les élèves. Un discours préliminaire leur faisait croire que la caméra ne se mettrait en marche qu'au bout d'une demi-heure de cours. Ce petit mensonge, déontologiquement condamnable, mais empiriquement fort efficace, nous a permis d'appréhender les comportements les plus spontanés possibles. De plus, pour que les élèves tendent à oublier le dispositif vidéo, nous avons placé la caméra le plus souvent sur le côté de la classe et plutôt au fond, évitant par là même qu'elle soit sous le regard constant des élèves.

6 L'enquête de terrain s'est déroulée durant les années scolaires 1987-1988 et 1988-1989. Nous avons recueilli, dans six collèges d'Aix-en-Provence sur les sept existants, 55 heures d'enregistrement dans 55 classes de sixième et cinquième. 700 élèves ont été observés, 358 filles et 342 garçons, dont l'âge varie le plus souvent entre 11 ans et 13 ans. Outre l'observation en classe, les élèves ont répondu à un questionnaire qui a notamment permis de définir leurs caractéristiques scolaires et sociologiques ${ }^{2}$.

7 L'observation vidéo, si elle facilite le recueil des données, pose le problème du traitement de l'information recueillie. Devant la richesse des images et des comportements, que le sociologue peut visionner autant de fois que nécessaire, une lecture à l'œil nu, qualitative, reste une gageure. On risque, dans ce cas, de mettre en œuvre les catégories de perception propres au chercheur (et, pourquoi pas, à son humeur du jour) sans en expliciter les fondements. Il nous fallait, en effet, rendre compte de manière systématique des différences de comportements selon les variables définies, par hypothèse, comme déterminantes. De ce fait, nous avons choisi d'établir une grille de codage qui rend compte des comportements observés de manière constante. Définir une grille d'observation, c'est définir comme pertinent un certain nombre de traits comportementaux : faut-il retenir, comme aurait pu le faire R. L. Birdwhistell (1984), dans une approche kinésique, le mouvement presque imperceptible de l'index sur le stylo, ou les variations de la position du sourcil de tel ou tel élève? Dans ces conditions, le travail 
de codage aurait nécessité des années de travail pour des résultats incertains. Nous avons donc choisi de définir le niveau de pertinence des comportements du point de vue de l'institution: le chahut, défini comme «tous cas de désordre qui résulte directement ou indirectement d'un acte positif ou négatif, accompli par l'élève lui-même et qu'il savait devoir produire ce résultat » (Testanière, 1967a), implique la visibilité de l'acte accompli, car il ne peut y avoir désordre si personne ne s'aperçoit de l'acte de chahut. De ce point de vue, un élève qui se lève en plein cours pour discuter avec un autre élève adopte un comportement pertinent et visible par le professeur, s'il se gratte l'oreille nous pensons que la pertinence de ce comportement est moindre du point de vue de notre objet.

Il va sans dire que nous ne pourrons appréhender que les comportements manifestes des élèves, et que certaines ambiguïtés ne peuvent être levées. Des formes particulières du chahut ne pourront être prises en compte dans notre analyse. Prenons l'exemple du chahut qui consiste à jouer l'élève hyper-sérieux, et ainsi interrompre le bon déroulement du discours professoral par des questions incessantes et plus ou moins en rapport avec l'objet du cours. Notre grille de codage ne pourra prendre en compte cette dimension particulière de perturbation de la communication pédagogique.

Notre grille de lecture des comportements s'inspire du travail de B. Zazzo (1976) sur les comportements à l'école maternelle. Nous sommes parti du recensement exhaustif des comportements possibles de chahut à partir des bandes vidéo recueillies, et ainsi recensé cinq manifestations possibles du chahut. D'abord le bavardage avec un voisin de banc pour des motifs autres que le travail en groupe. La difficulté réside ici dans la définition du but de l'interaction pour distinguer l'interaction dans le travail (alors codé comme une forme de participation à la classe) et l'interaction hors travail (qui correspond à une forme de chahut). Nous avons, pour départager ces comportements proches extérieurement, utilisé le son, puisque le micro de la caméra a permis de saisir ce que disaient les élèves. Mais ceci n'a pas toujours été possible, le son étant souvent noyé dans un bruit de fond perturbateur. La différenciation entre le bavardage et la participation entre pairs s'est faite, dans la plupart des cas, par les gestes adoptés lors de l'interaction : dans le cas de sourires, et de regards furtifs et méfiants vers le professeur, nous avons classé le comportement en question comme une forme de chahut; si, en revanche, l'interaction est suivie de regards vers le tableau, de lecture du cahier du voisin, ou de l'écriture d'une phrase sur le cahier, nous ne l'avons pas codée comme une forme de chahut, mais comme une forme de participation en classe. Les cas indécidables ont été codés en participation (la présomption d'innocence étant la règle). Il va sans dire que l'élève assez malin et expérimenté pour bavarder avec son camarade de banc tout en donnant l'impression de travailler ne peut être décelé par l'observateur : rappelons que nous n'appréhendons que les formes extérieures des comportements, ce qui peut laisser échapper quelques cas. Les autres comportements de chahut sont plus facilement identifiables : les grands gestes, les grimaces (la plupart du temps vers la caméra), les rires bruyants qui tendent à déranger le déroulement normal de la classe ont été codés sous l'appellation « grimaces, gestes, rires ».

10 Le chahut se manifeste aussi par des déplacements à l'intérieur de la classe sans rapport avec le travail ("déplacements illicites»), ou pour aller bousculer un autre élève ("bousculades, bagarres »). Nous avons classé comme une forme de chahut les échanges d'objets sans rapport avec le travail: des bandes dessinées par exemple, des jeux électroniques ou un baladeur. Enfin, un indicateur global de chahut a été construit par synthèse des cinq manifestations possibles relevées dans la grille de codage. 
Chaque individu a été codé pendant 20 minutes, puis nous avons calculé la proportion de chaque item par rapport à la totalité des comportements adoptés : ainsi, nous considérons qu'un élève chahute si au moins $5 \%$ de ces comportements sont classés comme une forme de chahut. Le seuil a donc été placé relativement bas, ce qui implique les pourcentages élevés des tableaux 2 et 3. Ceci a été rendu nécessaire par la rareté des comportements de chahut par rapport aux autres comportements. Nous en avons pour preuve le tableau 1 :

Tableau 1. Indicateur global de chahut (\% en ligne)

\begin{tabular}{lcccc}
\hline & $\begin{array}{c}\text { Seuil } \\
\text { <a } 5 \%\end{array}$ & $\begin{array}{r}\text { Seuil } \\
5 \%\end{array}$ & $\begin{array}{r}\text { Seuil } \\
10 \%\end{array}$ & $\begin{array}{c}\text { Seuil } \\
20 \%\end{array}$ \\
\cline { 2 - 5 } FILLES & $41 \%$ & $58 \%$ & $1 \%$ & $0 \%$ \\
\cline { 2 - 5 } GARCONS & $19 \%$ & $73 \%$ & $8 \%$ & $0 \%$ \\
\hline ENSEMBLE & $30 \%$ & $65 \%$ & $5 \%$ & $0 \%$ \\
\hline
\end{tabular}

12 Le chahut reste rare et marginal, ceux qui chahutent souvent (seuil $10 \%$ ) ou très souvent (seuil $20 \%$ ) sont l'exception et non la règle. Ne nous laissons donc pas impressionner par le $81 \%$ de chahut des garçons du tableau 3 : ce chiffre n'a pas de véritable sens en luimême et ne doit être interprété que par comparaison au $59 \%$ correspondant au chahut des filles (tableau 2).

\section{Les comportements de chahut des filles et des garçons}

13 La mesure des différences de taux de chahut ne peut prendre en compte le sexe comme seule variable déterminante. C'est en effet dans son articulation à la variable de l'origine sociale que nous pourrons rendre pleinement compte de l'effet spécifique du sexe sur le chahut en classe. Les deux variables sont inséparables, J.-C. Passeron et F. de Singly (1984) le soulignent très bien dans leur article pionnier en la matière.

«Il est clair [...] que la sociologie doit mettre en œuvre dans ses descriptions le double principe qui commande, de manière presque toujours croisée, dans les rapports d'inégalité comme dans leur redoublement symbolique, les oppositions et les spécifications qui tiennent simultanément à la différence de sexe et à la différence de classe. »

14 C'est aussi dans son articulation aux performances scolaires ${ }^{3}$ que nous pourrons pleinement rendre compte de notre hypothèse de départ : peut-on expliquer la meilleure réussite scolaire des filles par leur plus grande maîtrise, ou leur plus grand respect des codes interactifs de l'espace de la classe? Enfin, nous avons pris en compte une variable contextuelle qui donne une bonne mesure de l'aspect interactif des comportements en classe : le fait que le professeur soit un homme ou une femme semble en effet pertinent pour rendre compte des comportements de refus de l'école que sont les comportements de chahut et montrent bien ce que doivent aux rapports sociaux de sexe les comportements des élèves en classe. 
Tableau 2. Filles. Comportements de chahut en classe, selon le milieu social, les performances scolaires et le sexe du professeur (en \%)

\begin{tabular}{|c|c|c|c|c|c|c|}
\hline & $\begin{array}{l}\text { Indicat } \\
\text { globa! } \\
\text { de chahut }\end{array}$ & $\begin{array}{l}\text { Bavardago } \\
\text { avec son } \\
\text { voisin }\end{array}$ & $\begin{array}{l}\text { Grimaces } \\
\text { gestes } \\
\text { ries }\end{array}$ & $\begin{array}{l}\text { Bousculad } \\
\text { bagarres }\end{array}$ & $\begin{array}{l}\text { Depla. } \\
\text { cernents } \\
\text { llicite }\end{array}$ & $\begin{array}{l}\text { Echanges } \\
\text { dobiets }\end{array}$ \\
\hline Ensemble & 59 & 53 & 10 & 0 & 0 & 0 \\
\hline Ouvrier & 56 & 48 & 11 & 1 & 0 & 0 \\
\hline Irtem & 65 & 62 & 8 & 0 & 1 & 0 \\
\hline Cadre & 53 & 53 & 13 & 0 & 0 & 0 \\
\hline Bons & 55 & 51 & 8 & 0 & 0 & 0 \\
\hline Moyens & $6 B$ & 58 & 20 & 0 & 0 & 0 \\
\hline Maurais & 67 & 57 & 12 & 0 & 0 & 0 \\
\hline Prol homme & 48 & 42 & 0 & 0 & 0 & 0 \\
\hline Prol lemme & 63 & 58 & 14 & 0 & 0 & 0 \\
\hline
\end{tabular}

Tableau 3. Garçons. Comportements de chahut en classe, selon le milieu social, les performances scolaires et le sexe du professeur (en \%)

\begin{tabular}{|c|c|c|c|c|c|c|}
\hline & $\begin{array}{l}\text { Indicat } \\
\text { giotasi } \\
\text { de chahut }\end{array}$ & $\begin{array}{l}\text { Bavardage } \\
\text { avec son } \\
\text { voisin }\end{array}$ & $\begin{array}{l}\text { Grinaces } \\
\text { gestes } \\
\text { nies }\end{array}$ & $\begin{array}{l}\text { Bousculad } \\
\text { bagantes }\end{array}$ & $\begin{array}{l}\text { Dépla- } \\
\text { cements } \\
\text { illicto }\end{array}$ & $\begin{array}{c}\text { Echanges } \\
\text { dobjet }\end{array}$ \\
\hline Ensemble & 81 & 77 & 29 & 2 & 4 & 1 \\
\hline Orvinet & 78 & 75 & 31 & 0 & 3 & 0 \\
\hline Interm & 83 & 78 & 31 & 3 & 3 & 0 \\
\hline Cadre & 82 & 79 & 29 & 3 & 4 & 1 \\
\hline Bons & 81 & 76 & 30 & 3 & 1 & 3 \\
\hline Moyens & 81 & 79 & 23 & 2 & 0 & 2 \\
\hline Mauvais & 81 & 77 & 33 & 0 & 1 & 4 \\
\hline Prol homme & 78 & 74 & 17 & 2 & 0 & 0 \\
\hline Prot temme & 82 & 77 & 33 & 2 & 5 & 1 \\
\hline
\end{tabular}

\section{Le chahut est un phénomène masculin}

L'analyse comparative des tableaux 2 et 3 montre que les comportements de chahut en classe distinguent très fortement les filles des garçons : les garçons chahutent beaucoup plus souvent que les filles ( $81 \%$ contre $59 \%$ ). C'est le bavardage entre voisins de banc qui est la forme la plus répandue du chahut, c'est aussi, semble-t-il, la mieux acceptée par les professeurs qui peuvent, sans atteinte trop flagrante à leur autorité pédagogique, tolérer un bruit de fond. Inversement les formes les plus extrêmes du chahut, comme se déplacer en plein cours, gesticuler ou faire des grimaces représentent des ruptures trop vives et voyantes des régies interactives qui régissent la communication pédagogique pour être acceptée: dans ce cas le professeur se doit, sous peine d'atteinte à son autorité professorale, de rétablir le déroulement normal du cours en réprimandant - ou en excluant - l'ostrogoth récalcitrant. Le phénomène du chahut, du bavardage aux bravades 
les plus extrêmes, est donc essentiellement masculin : pour le bavardage, l'écart fillesgarçons est de $24 \%$ et de $19 \%$ pour les grimaces, les grands gestes et les rires.

Nous pouvons illustrer la monopolisation des formes les plus extrêmes du chahut par les garçons dans les réactions devant la caméra, car la présence du matériel vidéo dans la salle de classe n'a pas manqué de susciter diverses réactions. Lors de l'installation de la caméra dans la classe au début du cours, nous avons observé des différences notables de réaction devant l'objectif : les seuls à faire des gestes vers la caméra, à coller leur visage sur l'optique ou à faire des grimaces étaient des garçons, alors que les filles, plus distantes, observaient en silence et manifestaient leur intérêt par des regards. Une des réactions à la situation d'enquête les plus intéressantes du point de vue méthodologique s'est produite en cours d'art plastique. Les élèves avaient la possibilité de se déplacer pour aller chercher de l'eau, ce qui nécessitait un passage devant la caméra. Deux garçons se sont arrêtés devant l'objectif pour faire des grimaces: tirer la langue avec des yeux exorbités, se déformer les joues avec les doigts pour paraitre laid, faire des gestes obscènes et de défis... Une seule fille s'est postée devant l'objectif, mais sa réaction fut tout autre: elle se coiffa, en utilisant l'objectif comme un miroir, enleva ses lunettes, ramena ses cheveux en arrière pour mettre en valeur son visage. Après un demi-sourire elle continua son chemin vers sa place.

Ces deux réactions opposées devant la caméra, aussi anecdotiques soient-elles, montrent des relations différentes à l'image de soi et au corps pour les filles et les garçons. Certes de tels comportements ne se seraient pas manifestés sans la présence du dispositif d'enquête. Mais c'est peut-être là l'intérêt de l'observation : dans ce cas précis, la caméra joue un rôle de catalyseur qui permet d'éclairer des différences de comportements, elle entraine une perturbation qui fait partie intégrante de l'observation et qui révèle des différences pertinentes du point de vue de l'objet étudié. Le rapport au corps et à l'image de soi qui organise les réactions des élèves semble montrer un penchant, chez les garçons, à manifester ostentatoirement leur présence, qui se retrouve dans les comportements de chahut observés pendant les cours. Le milieu social d'origine ne fait que faiblement varier le phénomène chez les garçons, ce qui tend à montrer que l'appartenance de sexe prend ici le pas sur l'appartenance sociale.

\section{De fortes variations selon l'origine sociale chez les filles, plus faibles chez les garçons}

C'est bien une homogénéité du taux de chahut selon l'origine sociale qui apparaît chez les garçons : les faibles variations, quel que soit l'indicateur considéré, montrent qu'ici le fait d'être un garçon est plus important que d'être cadre ou ouvrier.

Mais la question du sens du chahut se pose alors à l'observateur : le chahut ne prend-t-il pas tout son sens dans sa relation aux autres comportements possibles au sein de la classe? Et ne peut-on pas penser que le chahut des garçons de milieu ouvrier et cadre recouvre une réalité bien différente, une relation à l'école et aux normes scolaires bien différentes? Pour répondre à cette question, il nous faut introduire un autre type de comportement : la participation en classe. Construit sur le même modèle que le chahut, la participation en classe (lever le doigt pour se faire interroger, répondre ou poser une question, travailler entre pairs) est le signe d'une grande maîtrise des exigences de l'institution scolaire. Fortement valorisée par les professeurs, elle est le signe d'une forte intériorisation des valeurs de compétition et de travail qui caractérise l'école. Nous nous 
demanderons si le chahut généralisé des garçons selon l'origine sociale correspond dans tous les cas au même phénomène social, celui d'un rejet de l'école. Ne peut-on penser, comme le fait J. Testanière (1967b), que le chahut des cadres puisse correspondre à l'expression d'une distance cultivée vis-à-vis de l'école et de ces valeurs, et d'un véritable rejet chez les garçons de milieu ouvrier? Le graphique 1 montre cette différence de nature entre le chahut des garçons ouvrier et cadre :

Graphique 1. Comportements de chahut et de participation (Nuage de points et droite de régression)

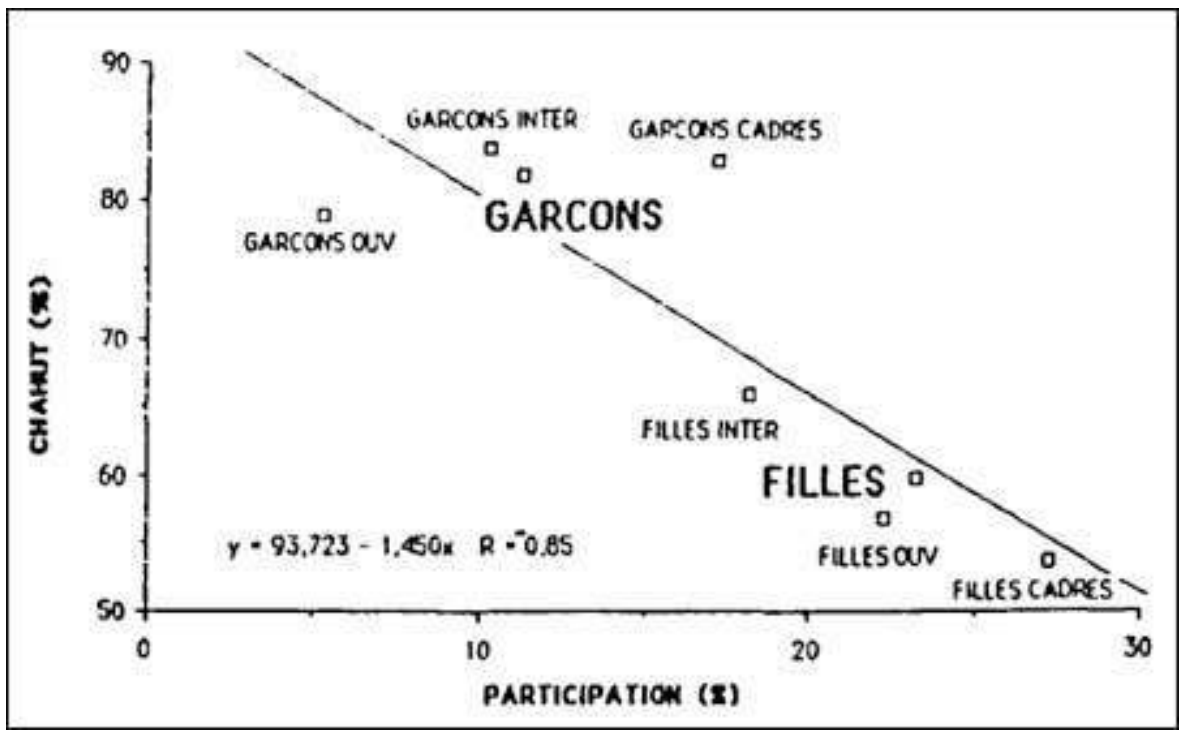

Le contraste entre les garçons de milieu ouvrier et cadre est ici marquant: du côté des cadres, le chahut est accompagné des comportements les plus valorisés par l'école, participer en classe et chahuter en même temps, c'est montrer à la fois des compétences scolaires, une forte intériorisation des valeurs de compétition de l'école, et une certaine distance à ces valeurs. Lorsque les garçons de milieu cadre chahutent, c'est pour dépasser des règles qu'ils ont fortement intériorisées et qu'ils peuvent se permettre de transgresser. Il en est tout autrement pour les garçons de milieu ouvrier : leur fort taux de chahut ne s'accompagne pas de comportements de participation en classe. Leur chahut semble l'expression d'un désarroi, d'une non-connaissance des règles implicites qui régissent l'espace de la classe, et d'une forte difficulté à intérioriser les valeurs scolaires qui leur sont trop étrangères pour être acceptées.

21 Les filles réagissent tout autrement dans leurs comportements : on ne voit pas chez les filles de milieu cadre ce chahut de défi qui caractérise leurs homologues garçons. On ne voit pas non plus chez les filles de milieu ouvrier de chahut qui exprimerait un quelconque rejet de l'école.

22 Ainsi, des comportements qui résultent d'une socialisation de sexe, valorisant l'affirmation de soi et le non-respect des règles, prennent un sens différent selon qu'ils s'articulent à un capital culturel plus ou moins important. Du côté des cadres, nous trouvons un chahut qui marque une distance cultivée aux règles interactives de l'espace scolaire. Du côté des ouvriers, nous trouvons plutôt le rejet d'un système vécu comme étranger et contraignant. La mise à distance qu'opère l'analyse statistique, si elle permet de rendre compte des relations objectives qui régissent les pratiques des sujets, fige quelque peu la diversité et la richesse des comportements qu'un observateur peut 
retrouver dans une salle de classe. Un retour aux données brutes s'impose donc pour rendre compte de cette diversité. Nous avons procédé à la sélection d'individus les plus représentatifs de leur catégorie par analyse factorielle des correspondances (de part leurs contributions et leurs coordonnées sur les deux premiers axes factoriels). Nous donnons ici, pour rendre compte des différences de chahut et de comportements entre les garçons de milieu ouvrier et cadre, l'observation approfondie et qualitative de Mathieu, fils de cadre et Joël, fils d'ouvrier.

Avec un fort taux de chahut (autant que les garçons mauvais élèves) les garçons de milieu cadre, la plupart du temps bons élèves, semblent manifester leur indépendance par rapport à l'institution, tout en mettant pleinement en œuvre leur capital culturel pour participer entièrement à la compétition scolaire au sein de la classe. Tout se passe comme si une certaine culture du chahut s'alliait aux comportements les plus conformes pour produire une sorte d'hurluberlu qu'illustre bien Mathieu, fils d'un cadre supérieur de l'administration et d'une professeur d'anglais. Ses résultats scolaires sont bons (13 sur 20 de moyenne générale au trimestre) sans pour cela être des meilleurs. Nous l'avons observé en mathématiques, où il est au premier rang. Le professeur est très interactif : il pose souvent des questions aux élèves tout en insérant de nouvelles connaissances en géométrie que les élèves doivent scrupuleusement noter sur leur cahier. Mathieu participe beaucoup à la classe : il lève souvent le doigt et ses réponses sont en accord avec ce qu'attend le professeur. Il ne pousse pourtant pas très loin sa fougue participative : de temps en temps on le voit s'abstraire du contexte de la classe pour se concentrer sur le travail à accomplir (recopier un schéma par exemple). Mais la conformité tranquille de ce bon élève est parfois rompue par des gestes vers la caméra ou des plaisanteries lancées à son voisin de banc lorsque le professeur ne le remarque pas. Son chahut, somme toute assez classique, reste clandestin et ne prend pas la forme d'un défi lancé au professeur : peut-être est-ce l'importance de l'enjeu scolaire des mathématiques qui produit là son effet.

Il en va tout autrement pour Joël, dont les comportements semblent aller plus dans le sens d'un refus généralisé des normes et valeurs scolaires. Joël, observé en cours de sciences naturelles, est fils de mineur, sa mère est sans profession, il a un frère et une sœur. Élève de $6^{\mathrm{e}}$, il a un an de retard et sa moyenne au trimestre est de 8,5 sur 20. C'est donc un mauvais élève dont les comportements sont en accord avec ses contreperformances scolaires. Les premières minutes de cours, il se tient relativement tranquille en écoutant le professeur expliquer un exercice. Mais il manifeste, de temps en temps, une certaine impatience qui prend les formes les plus diverses et qui peut facilement s'interpréter comme un défi : il interpelle le professeur en lui coupant la parole pour des motifs futiles, discute bruyamment avec un autre élève. Avec le temps qui passe, les comportements de Joël sont de plus en plus désordonnés : il écoute de moins en moins et commence à gesticuler pour mimer une chauve-souris (objet du cours) et faire rire ses camarades de banc qui ne s'en privent pas. Ils sont en effet quatre garçons au fond de la classe, et un certain effet de groupe se produit, chacun entraînant l'autre vers plus d'exubérance. Joël est parfois attentif, mais le temps joue contre lui : il ne peut rester concentré qu'un temps limité. Après dix minutes de cours, il va jusqu'à se lever de sa place pour discuter avec un autre élève, et ne se rassoit qu'après une réprimande du professeur, il gesticule vers la caméra avec ses trois complices dès que le professeur a le dos tourné. Le chahut est une composante essentielle des comportements de Joël. 
25 La mise en œuvre des valeurs de défi et d'affirmation de soi propres aux modèles masculins prend donc un sens différent selon qu'il s'articule à une hexis corporelle cadre ou ouvrière. Cependant dans les deux cas, l'affirmation de soi propre aux modèles masculins génère un chahut qui marque une non-maîtrise ou un non-respect des codes comportementaux qui régissent l'espace scolaire.

\section{Les performances scolaires n'influent pas sur le chahut des garçons}

La relation constatée à propos de l'origine sociale se retrouve ici pour les performances scolaires des filles et des garçons. Dans la population des garçons, bon élève ne veut pas dire élève non chahuteur. Au contraire, ils sont parmi les plus actifs lorsqu'il s'agit de bavarder ou de grimacer. Les indicateurs plus rares vont d'ailleurs dans le même sens : pour les bousculades ou les déplacements illicites, les bons élèves ne sont pas les derniers! Pour les filles, le taux de chahut varie avec les performances scolaires. Les principes de variations du chahut des filles sont moins inattendus que ceux des garçons : leur chahut est rare, il est d'autant plus important que les performances scolaires sont mauvaises et prend les formes les plus acceptables comme le bavardage.

La relation entre maîtrise des codes comportementaux et réussite scolaire semble donc difficile à établir pour les garçons. Ils développent en effet une culture du chahut qui masque la relation bien établie pour les filles. Tout se passe comme si le moindre chahut des filles, et donc leur meilleure maîtrise des codes interactifs de la classe, leur permettait une rentabilisation de la communication pédagogique bien supérieure aux garçons. Par la mise en œuvre de compétences spécifiques, acquises au sein du milieu familial, les filles font preuve d'une maîtrise de l'interaction scolaire qui leur permet d'obtenir des résultats bien supérieurs à ceux des garçons. Inversement, les attitudes de défi et de refus observées pour les garçons constituent un handicap majeur, tout au moins au niveau de scolarisation considéré, qui implique leur sur-sélection par rapport aux filles.

Si le chahut des élèves dépend de leur sexe et de leur origine sociale, il n'est pas non plus indépendant des caractéristiques du professeur. Celui-ci est un acteur essentiel de l'interaction inhérente à toute communication pédagogique, et doit donc être pris en compte dans l'analyse.

\section{De l'autorité de l'homme}

Le fait que le professeur soit un homme ou une femme constitue une variable contextuelle de première importance. Les variations selon cette variable sont, en effet, très importantes, surtout chez les filles. Dans le cas d'un professeur homme, le chahut se tasse fortement pour ne prendre que les formes les plus acceptées comme le bavardage. Le fait de grimacer en plein cours ou de gesticuler pour se faire remarquer passe de $0 \%$ à $14 \%$ dans le cas d'un professeur femme chez les filles. Il passe de $17 \%$ à $33 \%$ chez les garçons. Les déplacements en cours sans autorisation ne sont le fait que des garçons avec un professeur femme, jamais les hommes n'ont à subir ce type de chahut qui remet largement en cause l'autorité du professeur et qui perturbe la communication pédagogique.

Cependant les différences de chahut selon le sexe du professeur sont plus importantes chez les filles que chez les garçons : pour l'indicateur global de chahut, l'écart est de $15 \%$ 
chez les filles et de $4 \%$ chez les garçons. Les filles sont donc plus sensibles que les garçons au fait que leur professeur soit un homme ou une femme.

31 Nous touchons peut-être ici l'un des points essentiels des rapports de sexe qui organisent, au moins en partie, la relation élève/professeur. Les formes les plus voyantes de chahut, celles qui représentent un défi envers le professeur (se déplacer pendant le cours, gesticuler, faire des grimaces, etc.) sont visiblement plus fréquentes avec une femme qu'avec un homme: de ce fait elles doivent être pensées dans le rapport plus général homme/femme : tout se passe comme si l'autorité, attribut socialement défini comme masculin, était moins légitimement reconnue à un professeur femme. Les filles ne sont d'ailleurs pas exclues de ce rapport : la non-reconnaissance de l'autorité des professeurs femmes est autant, et peut-être plus, le fait des filles que des garçons. Nous voyons se dessiner ici les rapports sociaux de sexe au travers des comportements des élèves. Même si le professeur, détenteur de l'autorité pédagogique, est d'abord le représentant de l'institution scolaire auprès des élèves, il est aussi un être sexué qui influe sur les comportements des élèves au travers des rapports de sexe socialement construits. Dans son travail sur la mixité dans l'enseignement secondaire. N. Mosconi (1989) montre bien que les professeurs, autant hommes que femmes, pensent leur autorité comme attribut du sexe masculin :

«Alors que pour les hommes, affirmer leur autorité revient à affirmer leur

"virilité", les femmes, au contraire, pour affirmer la leur, croient nécessaire de faire oublier leur féminité. Par leurs vêtements, l'apparence, l'attitude, surtout quand elles ont des classes de garçons, beaucoup d'entre elles s'efforcent de se faire aussi peu féminines que possible, voire de donner l'illusion de la virilité ou de la masculinité...»

Le fort chahut des garçons avec les professeurs femmes montre bien les limites de cette illusion de la virilité : comment accepter l'autorité d'une femme, fût-elle professeur, quand on est un futur représentant du " sexe fort ». Chahuter un professeur femme, c'est refuser non seulement les règles de l'institution scolaire, mais c'est aussi refuser de plier devant celles qui sont dominées dans les rapports sociaux de sexe. «Tout se passe comme si, malgré la mixité, la classe restait une "affaire d'homme", un monde dominé par des relations et des certitudes viriles" (Mosconi, 1989). C'est aussi les rapports de sexes qui conditionnent le tassement du chahut des garçons avec un professeur homme. La reconnaissance de l'autorité va de pair avec la reconnaissance de l'homme comme détenteur « naturel » du pouvoir.

\section{Chahut et réussite scolaire des filles et des garçons}

33 L'interaction scolaire est le fruit d'un rapport complexe entre origine sociale et rapports sociaux de sexe. Nous l'avons particulièrement vue à l'œuvre dans la moindre autorité attachée aux professeurs femmes. Il semble que le chahut des garçons, même s'il prend des formes différentes selon le milieu social d'origine, soit le fruit de la mise en œuvre des modèles attachés au sexe masculin. De même la maîtrise comportementale dont font preuve les filles trouve ses fondements dans la socialisation de sexe qui conditionne une hexis corporelle apte à satisfaire les exigences de la communication pédagogique, et ainsi à conditionner la meilleure réussite scolaire des filles par rapport aux garçons. 


\section{BIBLIOGRAPHIE}

BiRDWhistell, R. L. - «Un exercice de kinésique et de linguistique : la scène de la cigarette », in G.

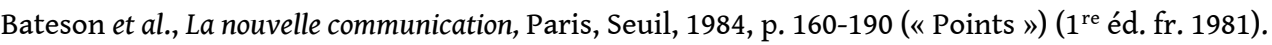
Bourdieu, P. \& PASSERON, J.-C. - La reproduction, Paris, Minuit, 1970 (« Le sens commun »).

CHERKAOUI, M. - Les changements du système éducatif en France 1950-1980, Paris, Presses universitaires de France, 1982 (« Sociologie)».

Establet, R. - L'école est-elle rentable ? Paris, Puf, 1987 (« Pédagogie d'aujourd'hui »).

FÉLOUZIS, G. - Filles et garçons au collège. Comportements, dispositions et réussite scolaires en $6^{e}$ et $5^{e}$, Aixen-Provence, Université de Provence-I, thèse de doctorat, 1990, multigr.

Mosconi, N. - La mixité dans renseignement secondaire : un faux semblant ?, Paris, PUF, 1989 («Pédagogie d'aujourd'hui »).

PASSERON, J.-C. \& SINGLY, F. de - « Différences dans la différence : socialisation de classe et socialisation sexuelle », Revue française des Sciences politiques, 34 (1), 1984, p. 48-78.

RAMOGNINO, N. - «L'observation, un résumé de la "réalité" : de quelques problèmes épistémologiques du recueil et du traitement des données », Cahier du CIRADE, Université du Québec à Montréal, 1989, p. 13-32.

TESTANIÈRE, J. - Désordre et chahut dans l'enseignement du second degré, Paris, 1967a, thèse, multigr. - «Chahut traditionnel et chahut anomique dans l'enseignement du second degré », Revue française de Sociologie, VIII, nº sp., 1967b, p. 17-33.

ZAzzo, B. - « Les conduites adaptatives en milieu scolaire : intérêt de la comparaison entre les garçons et les filles », Enfance, 4, 1982, p. 267-280.

\section{ANNEXES}

\section{Caractéristiques de l'échantillon observé}




\begin{tabular}{|c|c|c|c|}
\hline & & $\begin{array}{l}\text { FILLES } \\
n-358\end{array}$ & $\begin{array}{l}\text { GARCONS } \\
n=342\end{array}$ \\
\hline AGE & $\begin{array}{l}\text { N.A } \\
11 \text { ans } \\
12 \text { ans } \\
13 \text { ans } \\
14 \text { ans }\end{array}$ & $\begin{array}{r}5 \\
134 \\
143 \\
53 \\
23\end{array}$ & $\begin{array}{c}8 \\
105 \\
135 \\
70 \\
24\end{array}$ \\
\hline PCS & $\begin{array}{l}\text { Artcomm } \\
\text { Ouv/empl } \\
\text { Pro. Inter } \\
\text { Cadres }\end{array}$ & $\begin{array}{r}32 \\
137 \\
77 \\
108\end{array}$ & $\begin{array}{r}31 \\
128 \\
71 \\
112\end{array}$ \\
\hline CLASSE & $\begin{array}{l}\text { Soxieme } \\
\text { Cinquisme }\end{array}$ & $\begin{array}{l}188 \\
170\end{array}$ & $\begin{array}{l}153 \\
189\end{array}$ \\
\hline $\begin{array}{l}\text { PERF. } \\
\text { SCO }\end{array}$ & $\begin{array}{l}\text { BON } \\
\text { MOYEN } \\
\text { MAUVALS }\end{array}$ & $\begin{array}{r}233 \\
50 \\
75\end{array}$ & $\begin{array}{r}193 \\
62 \\
86\end{array}$ \\
\hline
\end{tabular}

Pour la définition de l'âge, nous considérons l'âge au 31 décembre de l'année en cours.

Pour définir l'origine sociale des élèves, nous avons pris en compte la profession du père et de la mère. Lorsque les deux parents travaillent, nous avons pris la position la plus élevée dans la hiérarchie sociale.

La population des filles est plus jeune que la population des garçons : les filles sont plus nombreuses que les garçons à avoir 11 ou 12 ans et elles sont moins nombreuses à être âgées de 13 et 14 ans. Ces différences peuvent s'expliquer par la plus forte proportion des filles observées en sixième (188 contre 153).

La distribution par catégories sociales est sensiblement égale entre les filles et les garçons. Cependant la faible représentation de la catégorie « artisans/commerçants » (36 filles et 31 garçons) ne nous a pas permis d'opérer des tris croisés dans cette catégorie qui ne figure pas dans nos analyses.

\section{NOTES}

1. Nous avons montré par ailleurs (Felouzis 1990) que d'autres éléments entrent en jeu pour expliquer la meilleure réussite des filles, et notamment les dispositions envers l'école, l'investissement scolaire et les ambitions professionnelles.

2. On trouvera en annexe la composition de l'échantillon observé.

3. La définition des bons et mauvais élèves s'est faite par la synthèse de deux indicateurs, l'âge et la moyenne obtenue au cours du trimestre. L'âge réel comparé à l'âge scolaire a permis la définition de trois groupes : les élèves en avance ou à l'âge légal ; les élèves avec un an de retard ; et les élève avec plus d'un an de retard.

La moyenne du trimestre durant lequel l'enquête s'est déroulée, a permis de définir cinq types de populations : ceux qui ont une moyenne inférieure à $8 / 20$ : ceux qui ont une moyenne comprise entre 8 et 9.9/20; ceux qui ont une moyenne comprise entre 10 et 11,9/20; ceux qui ont une moyenne comprise entre 12 et 13,9/20; et ceux qui ont une moyenne supérieure à 14 . 
La synthèse de ces deux indicateurs a permis de classer chaque élève en bon, moyen ou mauvais élève. 